\title{
Exploration of New England Native American Women's Views on Human Papillomavirus (HPV), Testing, and Vaccination
}

\author{
Deborah J. Bowen • Diane Weiner • Markos Samos • \\ Mary K. Canales
}

Received: 3 October 2013 /Revised: 16 December 2013 / Accepted: 8 January 2014 / Published online: 31 January 2014

(C) Cobb/NMA Health Institute 2014

\begin{abstract}
Objective Cervical cancer is a leading cause of mortality among Native American women. Cervical cancer is easily preventable with prescreening and follow-up. However, Pap smear screening rates are the lowest among Native women. This qualitative study assessed human papillomavirus (HPV) knowledge and acceptance of the HPV test and HPV vaccine for daughters among Native American women who reside on and off Native reservations in the Northeastern USA.

Design Our sample included 50 adult Native American female caregivers of Native American girls aged 9 to 18 years old. Five focus groups were conducted in the target region. Results Overall, participants had limited knowledge of HPV and the role of HPV vaccine to prevent cervical cancer incidence and mortality. Many women lacked knowledge about HPV, HPV test, and HPV vaccine; lacked consensus about the viability and safety of the HPV vaccine for Native American daughters; and were concerned that little information is known about the long-term effects of the HPV vaccine. The relationship with an Indian Health Service medical provider was strongly associated with HPV vaccine uptake and follow-up.
\end{abstract}

Diane Weiner is deceased.

D. J. Bowen $(\bowtie) \cdot$ D. Weiner

Department of Community Health Sciences, School of Public

Health, Boston University, 801 Massachusetts Avenue, Boston,

MA 02118, USA

e-mail: dbowen@bu.edu

M. Samos

Community Transition Program, United Community and Family

Services, 322 Main Street, Willimantic, CT 06226, USA

e-mail:msamos@ucfs.org

M. K. Canales

Department of Nursing, University of Wisconsin-Eau Claire, 105

Garfield Avenue, Eau Claire WI 54701, USA
Conclusions This qualitative study provides an important insight about Native women's perceptions related to preventive cervical cancer screenings, patient/provider communication, medical mistrust, and HPV vaccine acceptability. Health communication messages and cancer control interventions are needed that fully incorporate Native American culture, practices, and beliefs concerning health, illness, and healing.

Keywords Human papillomavirus · Vaccination · Native American

\section{Introduction}

Cervical cancer is the largest cause of early mortality among young women worldwide. American Indian/Alaskan Native (AI/AN) women are more likely to die from cervical cancer compared with white women http://seer.cancer.gov/statfacts/ $\mathrm{html} /$ cervix.html. The 5-year cancer survival rate from cervical cancer in 2010 also showed a disparity for AI/AN women (81\%) compared with white women (84\%) [1] (Table 1).

Cervical cancer is easily preventable with screening and follow-up. Cervical cancer screening rates are lower among AI/AN compared with white women [19, 23]. In 2008, the Pap test screening rates were $69.4 \%$ for $\mathrm{AI} / \mathrm{AN}$ women compared with $74.9 \%$ for white women, $81.1 \%$ for black women, and $75.4 \%$ for Hispanic women [4]. In 2003, Pap smear utilization within the past 3 years declined the sharpest among AI/AN women at $15 \%$ compared with US whites $21 \%$, Hispanics $25 \%$, and African Americans $16 \%$ [20]. Possible explanations for lower participation in cancer screening can be attributed to Native women's lack of health insurance, medical system distrust, misclassification of race [4], attitudes of fatalism about health [8], fear of cancer diagnosis [4], and spiritual beliefs about health [8]. 
Table 1 Major HPV-related themes

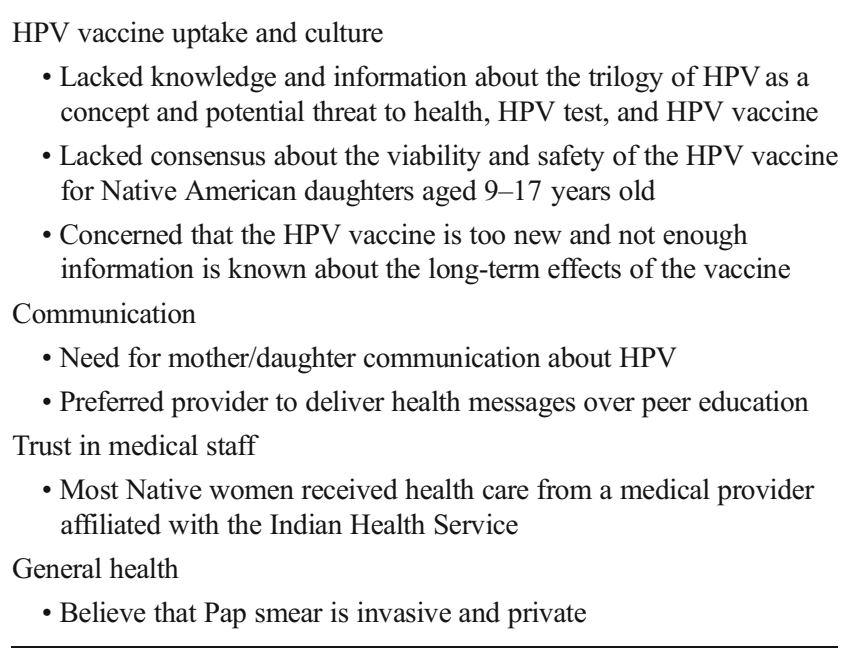

HPV, a sexually transmitted infection, has been identified as the major causal agent of cervical cancer [14]. While HPV infection is common among all races and age groups of women (estimated infection rates in the population are approximately $50 \%$ ), a higher prevalence has been found among women who are impoverished, undereducated, and belong to specific nonwhite racial/ethnic groups [9, 18]. In 2006, the Food and Drug Administration approved the three-shot vaccination HPV4, or Gardasil ${ }^{\circledR}$, for use in girls 9-18 years old to protect against HPV types 6, 11, 16, and 18, those most commonly associated with cervical cancer [21]. Currently, a commercially obtainable test for determining the presence of HPV is clinically available. However, the US Preventive Services Task Force concludes that the evidence is insufficient to recommend for or against the routine use of HPV testing as a primary screening test for cervical cancer [22].

Studies have examined HPV vaccine acceptability among white women [5] and college students [15]. The few studies involving Native participants have been mostly quantitative in nature [11, 12, 17], measuring prevalence and demographic predictors of vaccine uptake. Recently published data from Pacific Northwest and Plains tribes have identified lack of knowledge about the connection between HPV and cervical cancer and lack of financial resources to pay for vaccination as the two key barriers [3, 16]. No data have been published on eastern tribes' perspectives on HPV vaccination, and the limited data on Native American women suggest that there is variation among tribes in attitudes and knowledge about HPV and cervical cancer [3, 16]. This finding underscores the need for research documenting the views of Native women from multiple tribal and urban settings.
HPV4 Vaccine Utilization

The HPV4 vaccine remains underutilized among all ethnic minority women [6]. For HPV4, approximately half of adolescent girls had initiated the vaccination series in 2010, and approximately one fourth of the girls had received $\geq 3$ doses. White adolescent girls were more likely to complete three doses of HPV vaccine compared with Black or Hispanic adolescent girls. Empirical studies are needed to understand the motivations and address the barriers to vaccine uptake and protocol completion.

This study was conducted to gain insight into cancerscreening practices among American Indian women who reside in both a reservation and city to increase our understanding for cultural and environmental barriers related to HPV vaccine acceptability (intentions) and perceptions about cervical cancer screening.

\section{Methods}

\section{Participants}

Study participants were caregivers of adolescent Native American girls aged 9 to 18 years old and for whom the HPV vaccine is recommended. Caregivers were parents or any individual who was self-identified as being responsible for the child's health care. Parents/caregivers were adults, aged 18 to 64 years old, self-identified as Native American, and were English proficient. Participants were recruited from March 2009 through September 2010.

The study was conducted in the Northeastern Region of the United States, in tribes from six states: Maine, New Hampshire, Vermont, Massachusetts, Rhode Island, and Connecticut. There are approximately 3.1 million individuals in the USA who self-identify as AI/AN. The Northeast has a total of 71,999 mile $^{2}$ and a population of about $14,429,720$ people. $\mathrm{AI} / \mathrm{AN}$ individuals represent $1.37 \%$ of the US population, for a total of approximately 2.5 million in the Northeast. This study was reviewed and approved by the Institutional Review Board of the Boston University Medical Center.

\section{Recruitment}

We contacted the tribal leadership of multiple AI/AN tribes and nations in the Northeast to ask permission to hold focus groups on their land. Participants responded to recruitment flyers posted in public places, to word of mouth discussions among tribal leaders, and to referrals from social groups at tribal businesses. Flyers provided information about the purpose, benefits, and criteria for participation in the study. Contact information was given for interested women to alert researchers to their interest. 
Each participant was compensated \$20 for their participation in the study. This sum was paid to the participant at the end of the focus group. Each participant was also offered the cost of a round trip bus fare to defray the cost of transportation to attend the focus group. If a participant withdrew from the study after they arrived at the focus group but before they completed the focus group, they were still compensated for their time.

\section{Procedures}

Five focus groups were conducted, each in a different tribal community. The groups were held in community settings such as local libraries or community centers. Each group had between 6 and 17 participants, for a total of 102 participants. Sessions lasted about $90 \mathrm{~min}$ in duration. Informed consent was reviewed and obtained in writing prior to the start of each focus group. A trained female facilitator (an African American woman) introduced herself and explained the purpose of the study. To maintain confidentiality, she asked participants to introduce themselves using only first names or a pseudonym if they preferred. All sessions were digitally recorded and transcribed.

Using a pretested interview guide, the facilitator first asked the group members a series of questions about health and disease in general and how family members understood illness and disease. The questions then became more specific to cervical cancer, prevention of cervical cancer, Pap smears, and HPV infection, testing, and vaccination. Participants were asked to provide responses related to their current views and current behaviors about HPV infection, vaccine, and cervical cancer screening. Participants were then asked to provide suggestions for improving cervical cancer screening in their communities. After the end of the focus group, the facilitator handed the women a CDC brochure about cervical cancer, corrected any misperceptions that the women had identified during the focus group, and answered any questions that they had about cervical cancer or HPV.

\section{Data Analysis}

Audiotapes from the discussions were deidentified and transcribed. Data were coded, analyzed, and interpreted to identify emerging themes based on participants' responses. The analytic procedures that were used are informed by the general approach to qualitative coding, which necessitated an iterative research protocol with the goal to determine themes that capture the dimensions of Native women's experiences. Towards this end, two members of the study team jointly examined one transcript. The joint examination of one transcript served the purpose of developing a common set of content codes [13]. Thereafter, two study members independently applied these codes to an additional transcript. Once the second transcript had been coded independently, the two study members compared their coding to assess reliability. Discrepancies were resolved, and coding rules were modified through iterative discussion. This process required recoding of the first transcript using the modified coding rules. The final transcripts were coded by following the same process.

\section{Results}

During the coding process, several themes emerged from the focus groups that were relevant to the considerations of HPV testing and HPV vaccinations. These included ideas about prevention of the disease, importance and diverse roles of providers in improving health, confusion about HPV testing and vaccination, distrust of medical cures, and specific issues concerning HPV vaccination in daughters.

\section{Disease Prevention is Important}

One consistent message that most women in all groups echoed was the idea that taking measures to prevent disease was a good thing, and more women should engage in preventive behaviors. However, only a few of the women consistently engaged in preventive behaviors or commented that prevention was not important or preferable to treatment. Most women talked about getting regular mammograms, and although some women brought up negative experiences with mammograms, such as pain or embarrassment, all women were in agreement that mammograms saved lives and were a common and normal part of taking care of oneself. Most women expressed similar attitudes about getting a Pap smear, although a few women indicated that Pap smears were more private and more invasive. A few women indicated that they had a Pap smear during their last visit to a health care provider.

Facilitator: So what about getting Pap smears? Is that painful? Or do you...

P1: It's not painful. The Pap smear isn't painful, but I

think it's more intrusive...

P2: It's like a pride thing.

P1: Yeah more embarrassing.

HPV Vaccine Recommendations are Unclear

There was much discussion in all groups about the appropriate age of vaccination within the recommended range of 918 years old. Many women expressed the idea that 9 years old was too young to get a vaccine that protected against a sexually transmitted disease because the child was still a few 
years away from having sex, and it was at that later point that the decision should be made about vaccination or not. Some women pointed out that by the older age, it might be too late, and that if girls had to bring up a vaccine when they were considering having sex, it would operate the same as birth control in that if a child brings up birth control, it means that she is having sex and needs it. Participants both agreed and disagreed with this perspective and provided no clear resolution to this issue.

\section{Communicating with Daughter Varied}

Participants suggested that perhaps a good mother-daughter communication could eliminate or delay the need for the HPV vaccine, but others thought that good communication could simply allow for a mother to talk about the HPV vaccine with her daughters. Native women indicated that by the time that good mother-daughter communication about sexual issues occurred, it would be too late, as exposure would have already occurred. Some women indicated that the daughters were too young to have a say in whether they were to be vaccinated, but others felt like they had to ask their daughters and needed to put off the decision until the girls could make it for themselves.

P1: I left it up to the two oldest ones. I left it up to them.

Sat down, got as much information material as possible in regards to the whole HPV. Went through the family history with'em, between the aunts and both sides of the family and which ones have cancer so the likelihood. You know, so, the whole DNA thing.... So my daughter who's 17 years old now, she's a smart girl, I told her "this is your body and I'm not gonna to make that decision for you. Here's the information, you know, read up, when we go to the doctor you know, for the next time, talk with them, ask as many questions as you want, and then it's your judgment".

P1: When we sat down with $\mathrm{xxxx}$ (daughter), and I went through thing well why are they getting the shot,... I explained to her about the virus. Well how do you get it? I said 'well it's sexually transmitted' now she picked up - on - that and wanted to discuss it with me, and then I carry on. I think she, at $9 \mathrm{y} / \mathrm{o}$, she knows about sex... and so I explained to her about that. And, you know, I said you know 'you're 9 years old' you know. I said, you know I'm not gonna do it now, but when you get the shot, get the shot. She said 'No, I'll wait!' But I explained everything to her and you know, given her pediatrician explain it to her.

It seems to me that this vaccine, like when you see the commercials and all it's like "talk to your child". Well we don't have the opportunity to talk to our children about other things that we've had to give them. You know, it's like, it seems like a lot of people are saying it's their decision but in a way you know, it is up to the parent. Like you said, you can't bring them kicking and screaming, but if I felt that, if I felt so strongly about it, which I'm not sure that I do at this point, if I felt so strongly, yeah I'd bring 'em kicking and screaming, just like any other vaccine.

\section{Confusion about HPV Testing and HPV Vaccination}

Most women were confused about what HPV was, what an HPV test was, and what the HPV vaccine was meant to do. Many women confused the test with the vaccine or reported that they did not know that both were options. No one indicated that they thought HPV infection was related to human immunodeficiency (HIV) or AIDS. Most women did not know that HPV infection was so easily contracted, and very few women understood about different strains of virus carrying differential risk for disease.

Most women identified general concerns about prophylactic vaccines and possible risks associated with autism and other long-term health problems. For Native women, these health risks were perceived as real, and they had concerns about potential side effects associated with the HPV vaccine. A few women reported that one had to weigh the very real risks of vaccination against the small risks of cervical cancer for their daughters.

P1: It's, I mean, there are percentages, there's risk and everything, but that adult person, as to whether you want to take that risk, or you don't... or you run a risk of em getting the disease. That's what it comes down to me. It's the risk of the drug against the risk of the disease itself.

P1: You know, ... when they come to me, you know, I just, I don't like putting any foreign objects, substance in my body, that's man made...I don't know, I just can't, I can't do it. When it comes to the innovation shots with the kids, you know, some of them I think it's, like small pox. come on, people, ... why are you shooting my kid up with this live virus, what's the matter with you? Then the whole circumstances, you know, ...well gee, guess what? you have the innovation right there, so why put my kid, even though the risk factor's so low but, you know, they could be the 1 in you know 100,000 deaths or could come down with, now with some of the shots they are saying that it has to do with autism later in life. Participant-The mercury? I had a very hard time with that also. Especially with my son because it's, the rates are higher in boys. I had a really, I held off for longer than you're supposed to, because I just didn't feel comfortable. 
Patient-Provider Relationship is Important

Most women indicated that they had a private doctor or saw someone at the Indian Health Service (IHS) clinic affiliated with their tribe. They talked about the importance of having this source of good and trusted information.

$\mathrm{P} 1$ : And my primary care is at IHS. My gynecologist is also in my town, Dr. xx who I absolutely love. But any other further-I know. He's wonderful. He is just absolutely phenomenal. Makes you feel at ease and you know it's uncomfortable to go anyhow. He's wonderful. And any further testing I'll go to TRIBAL CLINIC. The walk-in clinic...

A few women mentioned that it was hard to know what information was accurate and balanced and that the provider's job was to help the patient sort through any misinformation or confusion so as to make informed choices about their health care.

The women talked about different types of providers as being important or useful for different reasons. Doctors were wanted if the problem was serious, but nurses and other support staff were needed for services related to procedures, side effects, and other questions and problems that came up in regular care.

P1: It depends on the relationship that you have with the $\mathrm{OB} / \mathrm{GYN}$. Then it, you know, it's fine. You sit down and talk about golf and kids, and so it's like, down there, just carrying on a conversation... Just like when the doctor's open you know, and honest with you, layman's terms you know, you know, the whole medical terminology layman's terms it's um, but it also depends on you too as an individual if I mean, you ... with your physician...

When asked whether women from the community could serve as peer providers or educators about HPV, there was a general positive reaction. Women, however, expressed concern that these peers would need backup of real professionals to respond to questions perceived as difficult or involved. The model of peer educators working door to door did not seem right to many of the women. When asked about the acceptability of having peer educators deliver health messages to Native women that they knew, the participants did not perceive peer educators as being effective, expressing concerns about being bothered, caught off guard, or peers arriving at inconvenient times of the day. Most women felt that it would be better to hold events at local clinics, centers, and houses and invite neighbors and family in to hear about health issues from peer educators.
P1: But something like that I would want to be informed about. But I don't think I would like it coming from anyone other than either an educator or a health care professional. I don't think I would like it coming from... P2: me?

P1: yeah, especially you. (laughter) I don't know, I just think those are the two people it should be either a health care professional or an educator, whether the...

P2: Somebody who's been trained?

P1: yeah.

P1: If somebody came knocking to my door and said: you know,... I want to talk to you about this. I wouldn't like it. Even if I knew them, even if I knew what they were doing. But if there was like an event, maybe like tonight...

Privacy was only expressed as a concern with potential peer educators by few participants, and as long as women were free to share or not, this concern did not seem large in the women's minds.

\section{Medical Mistrust}

Most women expressed concern that the HPV vaccine had not been on the market for many years, and therefore, the long-term effects were unknown. Participants asked why a new vaccine was being tried out among Native women first, but most women simply did not want to give the vaccine to their daughters until more about the long-term effects were known.

P1: It's a little bit off what you're just asking us. It goes back to vaccine versus screening, that sort of thing, you know what I mean? I think that because of the way I think, on a more natural level I don't trust drug companies, I don't trust most drugs, or any really. Um, vaccines have side effects and could cause, this is a new vaccine, we don't know what any long-term side effects are to it. So I think in that way, if I had a daughter that was that young now. I have, my daughter's 33, 34. But SHE has a daughter. I think I would go more for the screening and educating my child about how HPV is transmitted and not just HPV but other ... sexually transmitted diseases. I think we need to teach our children, especially our daughters, how to listen to their bodies, you know, pay attention to their bodies, take responsibility for that

P1: Like I know when my mother was pregnant with me they gave this drug to solve morning sickness and look what happened to a lot of the females that in my, you know, in my time what happened to them. 


\section{Discussion}

This qualitative study provides important information about Native women's perceptions related to preventive cervical cancer screenings, patient/provider communication, medical mistrust, and HPV vaccine acceptability for daughters. Native women perceived that the Pap test is beneficial to health, but few engaged in these preventive screening behaviors. Barriers to cancer control measures included the pain experienced during the mammogram or embarrassment. In a recent study, Brown and colleagues reviewed several factors associated with HPV testing uptake among an ethnically diverse group of women [2]. Similar to our results, Brown and colleagues found that embarrassment and lack of knowledge were the barriers to screening among Canadian college women. The quantitative studies from Native women of the Northern Plains also support the idea that knowledge deficits account for some of the differences between white and Native women in vaccination uptake. Empirical studies are needed among Native American women to explore this further and to test methods of increasing knowledge and awareness of the importance of HPV vaccine uptake with the goal of reducing cervical cancer incidence and mortality.

However, knowledge change alone will not likely address all the issues of the participants in this study. Mistrust was filtered through the conversation at many levels, including mistrust of vaccinations, drug companies, providers, and overall medical professionals. These levels of mistrust might be harder to overcome compared with misunderstandings of the role of HPV in cervical cancer. Mistrust in Native communities has roots in historical events that have shaped the current complex relationships with dominant culture medical providers, and these are likely to remain even if women understand the issues of vaccination more accurately. Experience with providers that do listen and understand issues faced by Native women might help here but will take time and repeated exposure.

Native American women reported positive relationships with their health care providers. Women reported that they would prefer to receive health information from their IHS providers over the lay health worker. This view is similar to a previous finding among Native American tribes in North Carolina [10]. However, a recently published survey of clinics serving Native women in the Pacific Northwest indicated that many of them did not regularly recommend HPV vaccination to their patients [7]. A provider recommendation for a cancer screening was viewed as important to women. However, there is distrust of the safety of HPV vaccines for both male and female children. Of note, women commented that they felt pressured to have their child vaccinated in order to attend public school even though the HPV vaccine is not mandated in any of the states where women live.
The peer health educator model has shown success as an effective approach to both recruit potential participants for health programs and deliver health messages. There are increasing numbers of health promotion studies using this community engagement approach [8]. Participants in this study had mixed opinions as to the use of peer educators to deliver health messages about HPV acceptability. Messer and colleagues described this intervention effect as an attenuator, which acts as a barrier to success [10]. Native women perceived that unscheduled door-to-door outreach was an interruption and would be ill timed. Native women preferred to get health information at a health clinic or a community event that involved information dissemination, as opposed to peer educators arriving at their home unannounced.

Many participants were confused by the differences between the HPV test and the HPV vaccine. They lacked knowledge about the HPV test and its overall purpose as technology used to detect specific high-risk HPV types. Some women expressed great mistrust in the HPV vaccine and prophylactic vaccinations in general as a preventive measure to eliminate vaccine preventable diseases in children. Most participants in this study believed that 9 years old was too young to have a child get a vaccine that would protect against sexually transmitted infections (STI). These parents may believe that preadolescent children are not having sexual intercourse at the age of 9 years old, even though published reports suggest that rates of child abuse and neglect among $\mathrm{AI} / \mathrm{AN}$ are higher than those of other racial/ethnic groups.

Because participants agreed that mother-daughter communication is important, there is an opportunity to reduce adolescent sexual risk behaviors [15] as part of a family intervention. Mothers and daughters would benefit from a structured sexual health education program that includes information about HPV etiology, prevention, and treatment. Heath promotion initiatives designed for Native American women could be developed within the cultural context of AI/NAs in New England.

Several elements of this study limit the generalizability. The facilitators were African American, and this crossethnic identification may have influenced the responses slightly. As this was a convenience sample, the findings cannot be generalized to other Native American groups. The women were self-selected, and the sample was relatively small. Findings suggest that there are several key barriers, including lack of knowledge and cultural beliefs, to be considered when developing cervical cancer prevention educational materials and interventions to increase HPV vaccine uptake among Native American women living in the Northeastern USA

Acknowledgments This study was supported by grants from the National Cancer Institute (CA124394) and the Centers for Disease Control and Prevention (5U48DP001922-02). 


\section{References}

1. American Cancer Society (2011) Health of American Indian or Alaska Native population. In: CDC Fast Facts, Cancer Facts and Figures, 2009-2010. http://www.cdc.gov/nchs/fastats/indfacts.htm . Accessed 30 Nov 2011

2. Brown L, Ritvo P, Howlett R, Cotterchio M, Matthew A, Rosen B, et al. Attitudes toward HPV testing: interview findings from a random sample of women in Ontario, Canada. Health Care Women Int. 2007;28(9):782-98. doi:10.1080/07399330701563061.

3. Buchwald D, Muller C, Bell M, Schmidt-Grimminger D. Attitudes toward HPV vaccination among rural American Indian women and urban white women in the Northern Plains. Health Educ Behav. 2013;40(6):704-11. doi:10.1177/1090198113477111.

4. Burhansstipanov L, Seals B, Satter D (2011) American Indian Alaska native racial misclassification, BRFSS and cancer data. Native American Cancer Research. http://natamcancer.org/handouts/data-misclassBRFSS_incid-mort 06-21-08_HND.pdf. Accessed 16 Dec 2013.

5. Cates JR, Brewer NT, Fazekas KI, Mitchell CE, Smith JS. Racial differences in HPV knowledge, HPV vaccine acceptability, and related beliefs among rural, southern women. J Rural Health. 2009;25(1):93-7. doi:10.1111/j.1748-0361.2009.00204.x.

6. Centers for Disease Control and Prevention (2011) National and state vaccination coverage among adolescents aged 13 through 17 yearsUnited States, 2010. http://www.cdc.gov/mmwr/preview/ mmwrhtml/mm6033al.htm?s_cid=mm6033a1_w. Accessed 26 Aug 2011.

7. Duvall J, Buchwald D. Human papillomavirus vaccine policies among American Indian tribes in Washington State. J Pediatr Adolesc Gynecol. 2012;25(2):131-5. doi:10.1016/j.jpag.2011.11. 012.

8. Fernández ME, Gonzales A, Tortolero-Luna G, Williams J, Saavedra-Embesi M, Chan W, et al. Effectiveness of Cultivando la Salud: a breast and cervical cancer screening promotion program for low-income Hispanic women. Am J Public Health. 2009;99(5):93643. doi:10.2105/AJPH.2008.136713.

9. Markowitz LE, Hariri S, Lin C, Dunne EF, Steinau M, McQuillan G, et al. Reduction in human papillomavirus (HPV) prevalence among young women following HPV vaccine introduction in the United States, National Health and Nutrition Examination Surveys, 20032010. J Infect Dis. 2013. doi:10.1093/infdis/jit192. http://jid. oxfordjournals.org/content/early/2013/06/18/infdis.jit192.

10. Messer L, Steckler A, Dignan M. Early detection of cervical cancer among Native American women: a qualitative supplement to a quantitative study. Health Educ Behav. 1999;26(4):547-62.

11. Moreland S, Engelman K, Greiner KA, Mayo MS. Papanicolaou testing among Native American and Hispanic populations. Ethn Dis. 2006;16(1):223-7.

12. Pandhi N, Guadagnolo BA, Kanekar S, Petereit DG, Karki C, Smith MA. Intention to receive cancer screening in Native Americans from the Northern Plains. Cancer Causes Control. 2011;22(2):199-206. doi:10.1007/s10552-010-9687-2.

13. Patton MQ (2002) Qualitative research and evaluation methods. SAGE, New York.
14. Peyton CL, Gravitt PE, Hunt WC, Hundley RS, Zhao M, Apple RJ, et al. Determinants of genital human papillomavirus detection in a US population. J Infect Dis. 2001;183(11):1554-64. doi:10.1086/ 320696.

15. Roberts ME, Gerrard M, Reimer R, Gibbons FX. Mother-daughter communication and human papillomavirus vaccine uptake by college students. Pediatrics. 2010;125(5):982-9. doi:10.1542/peds.20092888. http://pediatrics.aappublications.org/content/125/5/982.

16. Schmidt-Grimminger D, Frerichs L, Black Bird AE, Workman K, Dobberpuhl M, Watanabe-Galloway S. HPV knowledge, attitudes, and beliefs among Northern Plains American Indian adolescents, parents, young adults, and health professionals. J Cancer Educ. 2013;28(2):357-66. doi:10.1007/s13187-013.

17. Schumacher MC, Slattery M, Lanier AP, Ma K, Edwards S, Ferucci $\mathrm{ED}$, et al. Prevalence and predictors of cancer screening among American Indian and Alaska native people: the EARTH study. Cancer Causes Control. 2008;19(7):725-37. doi:10.1007/s10552008-9135-8. http://www.ncbi.nlm.nih.gov/pmc/articles/ PMC2574651/.

18. Smith JS, Brewer NT, Saslow D, Alexander K, Chernofsky MR, Crosby R, et al. Recommendations for a national agenda to substantially reduce cervical cancer. Cancer Causes Control. 2013;24(8): 1583-93. doi:10.1007/s10552-013-0235-8.

19. Steele CB, Cardinez CJ, Richardson LC, Tom-Orme L, Shaw KM. Surveillance for health behaviors of American Indians and Alaska Natives-findings from the behavioral risk factor surveillance system, 2000-2006. Cancer. 2008;113(5 Suppl):1131-41. doi:10.1002/cncr. 23727.

20. The Kaiser Family Foundation (2011) Percent of women age 18 and older who report having had a Pap smear within the last three years, by race/ethnicity. http://kff.org/womens-health-policy/state-indicator/ pap-smear-rate-by-raceethnicity/. Accessed 31 January 2011

21. U.S. Food and Drug Administration (2011) FDA licenses new vaccine for prevention of cervical cancer and other diseases in females caused by human papillomavirus - Gardasil, June 8, 2006. http:// www.fda.gov/NewsEvents/Newsroom/PressAnnouncements/2006/ ucm108666.htm. Accessed 23 Dec 2011

22. U.S. Preventive Services Task Force (2003) Screening for cervical cancer. http://www.uspreventiveservicestaskforce.org/uspstf/ uspscerv.htm. Accessed 16 Dec 2013.

23. Yu TC, Chou CF, Johnson PJ, Ward A. Persistent disparities in Pap test use: assessments and predictions for Asian women in the U.S., 1982-2010. J Immigr Minor Health. 2010;12(4):445-53.

\section{Key Messages}

The key messages of this paper are as follows:

Native women present a wide range of ideas and knowledge about HPV vaccination.

Preventing cervical cancer in daughters is important to Native women. Intervention with mothers to help remember to vaccinate daughters might be a good avenue to improve cervical health among Native women. 